\title{
Flujo de capitales y crecimiento económico en el Perú: 1950 - 2014 (uso de vectores autorregresivos y la cointegración)
}

Flow of capital and economic growth in Peru: 1950 - 2014

(autoregressive vectors and cointegration use)

Jorge Manrique Cáceres ${ }^{1}$ y Pierina Norabuena Trejo ${ }^{1}$

\section{RESUMEN}

Los flujos de capitales (de corto y largo plazo) han tenido significativa relación con el crecimiento económico en el Perú, a lo largo de un período de 64 años, divididos en períodos bastante diferenciados. La división del período de estudio ha respondido a los diferentes modelos económicos implantados en su momento. Un primer período abarca desde 1950 hasta 1970, con predominio de una economía primario-exportadora; un segundo período de 1971 a 1990, con prevalencia de una corriente económica de sustitución de importaciones y un tercer período de 1991 al año 2014, con el regreso de una tendencia que privilegia la economía primario-exportadora y de carácter extractivista, con gran dependencia de las exportaciones mineras, agroexportadoras y pesqueras. El objetivo de la investigación es determinar en qué medida los flujos de capitales de corto y largo plazo inciden en el crecimiento del producto bruto interno del Perú, en el período 1950 - 2014. Además, se ha incorporado al análisis de los datos, las técnicas econométricas del uso de vectores auto regresivos (VEC), del mecanismo de corrección de errores (MCE) y de la cointegración como aporte académico al estudio de temas económicos, los cuales han resultado satisfactorios.

Palabras clave: flujos de capitales; inversión extranjera directa; inversión bruta fija; inversiones de corto plazo; crecimiento económico.

\section{ABSTRACT}

Economic growth in Peru has been the result of capital flows (short-and longterm), over a period of 64 years, divided into sub-periods markedly different. The division of the study period has responded to different economic models

1 Universidad Nacional Santiago Antúnez de Mayolo. Huaraz, Perú. 
implemented at the time. In the first period (1950-1970) predominates primary export economy; during the second period (1971-1990) import substitution was the prevailing model, and in the third period (1991-2014), it establishes a primary export economy again, with high dependence on mining, agricultural, fishing exports.

Determining the impact of capital flows short - and long - term economic growth of Peru is the main objective of the research.

The analysis is based on the econometric technique of vector autoregressive (VAR), the cointegration method and the estimation of error correction mechanism (MCE), as an academic contribution to the study of economic problems, which have proved successful.

Keywords: capital flows; foreign direct investment; gross fixed investment; short-term investments; economic growth.

\section{INTRODUCCIÓN}

A partir de los primeros años de la década de los noventa, el Perú experimentó un considerable incremento en la entrada neta de capitales ${ }^{2}$, crecimiento que se ha mantenido en las últimas seis décadas, lo cual significó una reversión de la tendencia observada en los ochenta. Para una mejor comprensión de estas etapas, es necesario hacer un breve recuento del crecimiento económico del Perú, desde 1950 hasta la actualidad.

Figura 1. Variación porcentual del PBI: 1950 - 2014

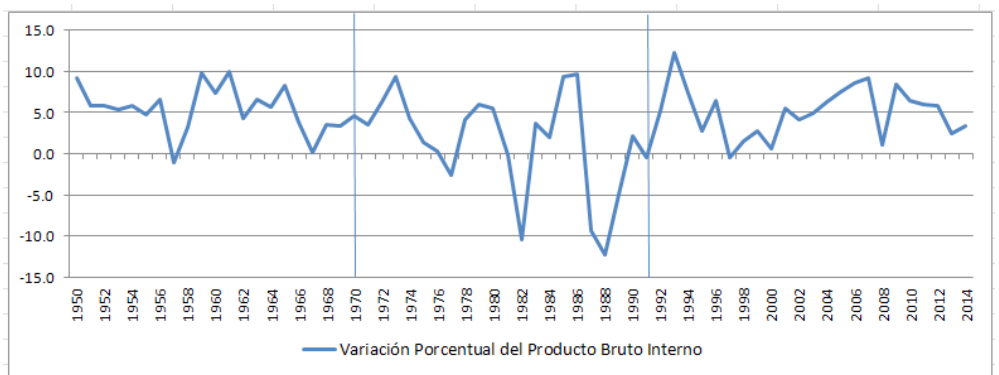

Fuente: Banco Central de Reserva del Perú (2014)

Como se muestra en el figura 1, en la evolución del crecimiento del PBI puede distinguirse tres etapas, tomando como referencia a Dancourt, Mendoza y

\footnotetext{
2 Se denomina flujo de capitales o entrada neta de capitales a la entrada total de fondos procedentes del extranjero menos la salida total de fondos nacionales que van al extranjero.
} 
Vilcapoma (1997) ${ }^{3}$, aunque con ligeras variaciones en los períodos. La primera etapa (1950-1975) es un período de rápido crecimiento, interrumpido por algunas recesiones breves (1958-1959; 1967-1969); el PBI crece a una tasa promedio de 5.5\% anual. La segunda etapa (1976-1990) se caracteriza como una crisis de larga duración con períodos violentos de recesión y auge. Esta segunda etapa es también un período de alta inflación que desemboca en la hiperinflación de 1988-1990. La tercera etapa (1991-2014) constituye un nuevo momento de estabilización y de expansión. Como resultado del programa de estabilización y las reformas aplicadas en la década de los noventa se produce una recuperación sustancial en el crecimiento del PBI. Este es un período de baja inflación y crecimiento continuo, interrumpido por algunas recesiones breves (1998-2001; 2009).

En cuanto a la evolución de los flujos de capitales (de corto y largo plazo), puede observarse que muestran un comportamiento diferenciado en estos tres períodos.

La figura 2 muestra la evolución de los flujos de capitales de largo plazo. En la primera etapa (entre 1950 y los primeros años de la década de los 70) se observa que la entrada neta de capitales de largo plazo a nuestro país fue relativamente baja y correspondió básicamente al sector público; se puede apreciar que a partir de mediados de la década de los sesenta la Inversión Directa Extranjera (IDE) tuvo una menor participación.

Figura 2. Evolución de los flujos de capital de Corto y Largo Plazo: 1950 - 2014 (millones de US\$)

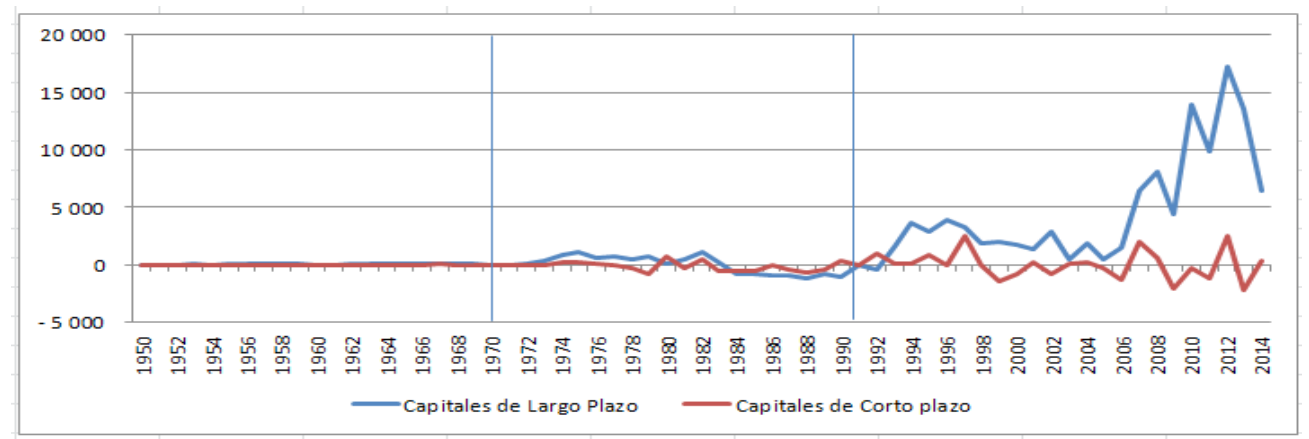

Fuente: Banco Central de Reserva del Perú (2014)

En la segunda etapa (entre los primeros años de la década de los setenta y noventa), la entrada neta de capitales de largo plazo empezó a adquirir mayor dina-

\footnotetext{
3 El flujo de capitales de largo plazo se desagrega entre el sector público y el sector privado. Este último está conformado básicamente por la Inversión Directa Extranjera (IDE).
} 
mismo. En los setenta continuó liderada por los capitales del sector público; sin embargo, en la década de los ochenta se aprecia una considerable caída tanto en el sector público como en el sector privado, a raíz de los problemas económicos que atravesaba nuestro país.

En la tercera etapa, tras la aplicación del programa de estabilización y un amplio programa de reformas estructurales a inicios de los noventa (que incluían entre otros aspectos la paulatina reducción del rol del Estado en la economía y la reforma del sistema financiero), se puede observar un considerable y continuo incremento en la entrada neta de capitales de largo plazo al Perú. En este período se produjo un cambio sustancial en la composición del flujo de capitales, ya que aumentó significativamente la participación de los capitales privados (a través de IDE) respecto a los capitales públicos.

Entre los antecedentes del presente trabajo podemos citar al desarrollado por Dancourt, Mendoza y Vilcapoma (1997), quienes sostienen que el patrón de las fluctuaciones económicas en el Perú parece ser independiente de los modelos de desarrollo o estrategias de crecimiento. Tanto en el período de vigencia del modelo de industrialización vía sustitución de importaciones, este patrón, sobre todo el de las recesiones, es el mismo. Las recesiones tienden a estar asociadas con una aceleración de las tasas de inflación y devaluación, una caída del gasto público y del créditos reales y, por último, con una caída de la capacidad de importación y una segunda conclusión es que no se abstrae el rol de los shocks externos cuando se busca explicar el comportamiento de la economía peruana durante 1950-96. De las seis recesiones identificadas en este período, todas, salvo una, coinciden con shocks externos adversos. También recurrimos al trabajo desarrollado por Cubillas (2013) quien afirma que los términos de intercambio y los flujos de capital de corto plazo son fuentes de choque por medio principalmente del sector minero y el sector financiero. Un choque negativo de los flujos de capital de corto plazo aumenta el costo de financiamiento de las instituciones financieras y reales que puedan acceder a financiamiento externo; se reduce la inversión.

La realización del presente trabajo tiene su justificación en dos aspectos principales; por una parte, explicar cuál ha sido el comportamiento de los flujos de capital que han llegado al país a partir de los años 50 a la fecha y cómo se ha comportado en función de los diferentes modelos económicos que se sucedieron a lo largo del período de estudio y, en segundo lugar, servir como referencia de un estudio econométrico, como parte de la labor docente. La aplicación de nuevas técnicas econométricas para explicar fenómenos económicos nos ha llevado a desarrollar este trabajo.

Los objetivos que el estudio se plantea son la de determinar en qué medida los flujos de capitales de corto y largo plazo inciden en el crecimiento del producto bruto interno del Perú, en el periodo 1950 - 2014, poniendo especial énfasis en el comportamiento de la IED y su incidencia en la evolución del producto nacional. 
Dentro de todo este escenario nacional podemos intentar una respuesta anticipada en los términos siguientes, los flujos de capitales de corto y largo plazo (compuestos por la inversión pública y la inversión privada (IDE), inciden positivamente en el crecimiento del producto bruto interno del Perú, en el período $1950-2014$.

Flujos de capital, Inversión Directa Extranjera y Crecimiento

En relación a la conveniencia o no de la IED, en los países subdesarrollados se tiene puntos de vista, bastante disímiles; por ejemplo: Álvarez, Barraza y Legato (2009:117), señalan:

La relación teórica entre Inversión Extranjera Directa y Crecimiento Económico difiere de acuerdo al marco de análisis utilizado. Los modelos de crecimiento neoclásicos implican que la Inversión Extranjera Directa no afecta al Crecimiento Económico en el largo plazo, como resultado de suponer mercados de competencia perfecta, productividad marginal decreciente y retornos constantes a escala. De este modo, la única manera de afectar el Crecimiento Económico en el largo plazo es a través de modificar dos factores exógenos: la tecnología y el trabajo... En contraste, los modelos de crecimiento endógeno indican, en general, que la Inversión Extranjera Directa tiene un efecto positivo en el Crecimiento Económico de manera indirecta a través tanto de la Formación de Capital como del desarrollo de los recursos humanos.

Mody y Murshid (2002) también citados por Boyan y Loza (2005), sugieren que la IDE tiene un impacto importante y significativo en la inversión nacional, a diferencia de la inversión de cartera y los préstamos que han tenido un impacto modesto. En este sentido, la creciente dependencia de la IDE es positiva para los países en desarrollo, dado que los inversionistas suelen comprometer sus recursos por un mayor plazo y están más capacitados que los tenedores de deuda para tolerar la adversidad de corto plazo. No obstante, para atraer más capital e IDE es necesario que existan mejores políticas. Según el Banco Mundial (2001), donde la capacidad de absorción es baja (es decir, el manejo macroeconómico, infraestructura y capital humano) los beneficios de la IDE son prácticamente inexistentes.

\section{MATERIALES Y MÉTODOS}

En lo referente al tipo de investigación, fue aplicada, descriptiva y correlacional y de diseño no experimental y longitudinal. La población en estudio son las variables macroeconómicas tasa de crecimiento del PIB, Flujo de capitales privados (IED), flujo de capitales de corto plazo y el flujo de capitales públicos, en el período 1950 - 2014. Los datos usados son de frecuencia anual. Todas estas series están expresadas en valores corrientes dolarizados. 
Esta información es tomada del Banco Central de Reserva del Perú (BCRP, 2014). Se recopiló información estadística de las estadísticas anuales. Para el análisis y tratamiento de la información, se hizo uso de la econometría de series temporales, el análisis univariante de no-estacionariedad, técnicas bivariantes de cointegración sobre la base de modelos de vectores autorregresivos (VAR) y la modelización a través de mecanismos de corrección de error (VEC) y la cointegración. Se usó el programa Eviews 8.1, para el procesamiento de los datos.

\section{RESULTADOS}

El objetivo del análisis empírico es determinar en qué medida los flujos de capitales o entradas netas de capitales (considerando sus distintos componentes) inciden en el crecimiento económico del Perú.

Para las distintas estimaciones se ha tomado series anuales del Producto Bruto Interno (PBI), flujo de capitales de corto plazo (CCP) y flujo de capitales de largo plazo (CLP)1; este último conformado por los capitales de largo plazo del sector público (SPUB) y la inversión directa extranjera (IDE), para el período año 1950 al 2014. Los datos económicos se expresan en millones de dólares constantes.

Para el análisis del periodo 1950-2014, se toma en cuenta los grandes cambios estructurales que se dieron a partir de los primeros años de la década de los 70 y a inicios de la década de los 90 . Se considera tres subperiodos; el primero de 1950 a 1970; el segundo de 1971 a 1990 y, el tercero, de 1991 a 2014 debido a los significativos cambios que se produjeron en la magnitud y composición de las entradas netas de capital, como se analizó en el apartado anterior; asimismo, teniendo en cuenta los estudios realizados con anterioridad ${ }^{4}$ en los que se determina los años en que se produjeron los cambios estructurales.

Análisis de estacionariedad de las series:

Los resultados de la prueba de raíz unitaria Dickey-Fuller Aumentada (ADF) aplicada a las variables de estudio aparecen en las tablas 1, 2 y 3 para los períodos 1950-1970, 1971-1990 y 1991-2014, respectivamente.

Esta prueba nos indica que las variables analizadas no son estacionarias en los períodos de estudio. Fue necesario diferenciar una vez para obtener rechazo de la existencia de raíz unitaria, lo cual permitió concluir que todas las series tienen el mismo nivel de integración, todas son integradas de orden uno I(1) al nivel de confianza del $95 \%$.

${ }^{4}$ Como principal referencia se considera el trabajo de Dancourt, Mendoza y Vilcapoma (1997). 
Tabla 1. Test ADF: Período $1950-1970$

\begin{tabular}{ccccc}
\hline Serie & Estadístico t & Valor crítico (al 5\%) & p-value & $\begin{array}{c}\text { Orden de } \\
\text { integración }\end{array}$ \\
\hline$\Delta \log$ PBI & -3.773 & -3.052 & 0.0125 & $\mathrm{I}(0)$ \\
$\Delta$ CCP & -6.908 & -3.066 & 0.0000 & $\mathrm{I}(0)$ \\
$\Delta$ CLP & -5.363 & -1.960 & 0.0000 & $\mathrm{I}(0)$ \\
$\Delta \mathrm{IDE}$ & -4.045 & -3.030 & 0.0064 & $\mathrm{I}(0)$ \\
$\Delta$ SPUB & -5.951 & -1.960 & 0.0000 & $\mathrm{I}(0)$ \\
\hline
\end{tabular}

Tabla 2. Test ADF: Período 1971 - 1990

\begin{tabular}{ccccc}
\hline Serie & Estadístico t & Valor crítico (al 5\%) & p-value & $\begin{array}{c}\text { Orden de } \\
\text { integración }\end{array}$ \\
\hline$\Delta \log$ PBI & -3.314 & -1.961 & 0.0024 & $\mathrm{I}(0)$ \\
$\Delta$ CCP & -3.670 & -1.963 & 0.0011 & $\mathrm{I}(0)$ \\
$\Delta$ CLP & -4.073 & -1.961 & 0.0004 & $\mathrm{I}(0)$ \\
$\Delta \mathrm{IDE}$ & -4.044 & -1.963 & 0.0004 & $\mathrm{I}(0)$ \\
$\Delta$ SPUB & -4.357 & -1.961 & 0.0002 & $\mathrm{I}(0)$ \\
\hline
\end{tabular}

Tabla 3. Test ADF: Período $1991-2014$

\begin{tabular}{ccccc}
\hline Serie & Estadístico t & Valor crítico (al 5\%) & p-value & $\begin{array}{c}\text { Orden de } \\
\text { integración }\end{array}$ \\
\hline$\Delta \log$ PBI & -2.253 & -1.957 & 0.0264 & $\mathrm{I}(0)$ \\
$\Delta$ CCP & -4.326 & -1.960 & 0.0002 & $\mathrm{I}(0)$ \\
$\Delta$ CLP & -6.548 & -1.957 & 0.0000 & $\mathrm{I}(0)$ \\
$\Delta \mathrm{IDE}$ & -5.465 & -1.957 & 0.0000 & $\mathrm{I}(0)$ \\
$\Delta$ SPUB & -4.121 & -1.960 & 0.0003 & $\mathrm{I}(0)$ \\
\hline
\end{tabular}

Dado que todas las variables resultaron I(1) para cada una de las etapas señaladas a lo largo del período 1950 - 2014, se procedió a determinar si existe una relación estable a largo plazo entre ellas, es decir, si las variables están cointegradas. Para realizar este análisis se utilizó la metodología de Johansen-Juselius.

El test de Johansen ${ }^{5}$ es una prueba de máxima verosimiltud basada en modelos VAR y que descansa sobre el supuesto esencial de que los residuos se distribuyen de forma normal y no presentan problemas de autocorrelación ni heteros-

\footnotetext{
5 El test de Johansen permite comprobar, por un lado, la existencia de una relación estable a largo plazo o de cointegración y, por otro lado, estimar los parámetros cointegrantes (ajuste a largo plazo). Sin embargo, en el corto plazo puede haber desequilibrio y el término de error suele tratarse en este escenario como el "error de equilibrio".
} 
cedasticidad. Por tanto, previamente a su aplicación realizamos la estimación de un modelo VAR por MCO para cada uno de los subperíodos de estudio: 1950-1970, 1971-1990 y 1991-2014 con el fin de determinar, en primer lugar, el número óptimo de rezagos ${ }^{6} \mathrm{y}$, en segundo lugar, comprobar si sus residuos cumplen con las propiedades señaladas.

En la tabla 4 puede observarse los criterios de información utilizados y la estructura de rezagos óptima para cada período, que es uno para los tres periodos de estudio: 1950-1970; 1971-1990 y 1991-2014, de acuerdo con los criterios de información LR, SC y HQ.

Tabla 4. Determinación del rezago óptimo del VAR

\begin{tabular}{cccccc}
\hline \multirow{2}{*}{ Periodo } & LR & AIC & SC & HQ & Rezago \\
\hline $1950-1970$ & $63.641^{*}$ & $17.740^{*}$ & $18.337^{*}$ & $17.841^{*}$ & 1 \\
$1971-1990$ & $40.082^{*}$ & $29.440^{*}$ & $30.034^{*}$ & $29.522^{*}$ & 1 \\
$1991-2014$ & $72.496^{*}$ & 34.326 & $34.921^{*}$ & $34.466^{*}$ & 1 \\
\hline
\end{tabular}

*Indica el criterio según el cual, el retardo consignado en la última columna es óptimo.

LR: sequential modified LR test statistic (each test at 5\% level)

AIC: Akaike information criterion

SC: Schwarz information criterion

HQ: Hannan-Quinn information criterion

Bajo la estructura de rezagos óptima analizamos el comportamiento de los residuos del VAR estimado en cada uno de los períodos de estudio. La tabla 5 muestra un resumen de las pruebas realizadas a los residuos. En cada uno de los períodos analizados, la diagnosis del modelo nos lleva a aceptar que los residuos se distribuyen de forma normal y no presentan problemas de autocorrelación ni heteroscedasticidad.

Tabla 5. Análisis de los residuos del VAR

\begin{tabular}{cccc}
\hline Período & Ho: normalidad & $\begin{array}{c}\text { Tests (p-values) } \\
\text { Ho: no autocorrelación } \\
\text { de primer orden }\end{array}$ & Ho: homoscedasticidad \\
\hline $1950-1970$ & 0.491 & 0.174 & 0.208 \\
$1971-1990$ & 0.080 & 0.329 & 0.223 \\
$1991-2014$ & 0.886 & 0.174 & 0.161 \\
\hline
\end{tabular}

\footnotetext{
${ }^{6}$ La elección del número óptimo de rezagos es esencial, dado que será precisamente dicho número el que se utilizará en el test de Johansen como la base del cálculo del número de vectores de cointegración.
} 
En cada uno de los períodos, el test de normalidad (utilizando la covarianza de Cholesky) muestra que ni el componente de la curtosis ni el de asimetría resultaron significativos y que la probabilidad conjunta es mayor al 5\%, por lo que se concluye que los residuos son normales. Asimismo el test de autocorrelación LM (Multiplicador de Lagrange) aplicado al VAR estimado permite afirmar al nivel de confianza del 95\% que no existe autocorrelación en los residuos. Por su parte, el test de White (sin términos cruzados) nos indica que los residuos son homoscedásticos, ya que la probabilidad conjunta es mayor al 5\%.

Por tanto, en cada uno de los períodos considerados y bajo la longitud óptima de retardos, los residuos están bien comportados y, en consecuencia, podemos desarrollar el análisis de cointegración de Johansen, buscando la existencia de una relación estable a largo plazo entre las variables de estudio.

\section{Contrastación de las hipótesis}

En la tabla 6 se muestra los resultados del test de cointegración de Johansen. Este método sugiere dos estadísticos para determinar el número de vectores de cointegración: el estadístico de la traza y la prueba del máximo valor propio.

Tabla 6. Test de Cointegración de Johansen-Hipótesis general

\begin{tabular}{|c|c|c|c|c|c|c|c|}
\hline \multirow[b]{2}{*}{ Periodo } & \multicolumn{4}{|c|}{ Test de Traza } & \multicolumn{3}{|c|}{ Test de Máximo Valor Propio } \\
\hline & Rezagos & $\begin{array}{l}\text { Hipótesis } \\
\text { nula }\end{array}$ & $\lambda$ traza & $\mathrm{VC}(5 \%)$ & $\begin{array}{l}\text { Hipótesis } \\
\text { nula }\end{array}$ & $\lambda$ máx & $\mathrm{VC}(5 \%)$ \\
\hline \multirow{3}{*}{ 1950-1970 } & \multirow{3}{*}{1} & $\mathrm{r}=0$ & 37.79 & 29.80 & $\mathrm{r}=0$ & 27.38 & 21.13 \\
\hline & & $\mathrm{r} \leq 1$ & 10.41 & 15.49 & $r=1$ & 10.02 & 14.26 \\
\hline & & $r \leq 2$ & 0.39 & 3.84 & $r=2$ & 0.39 & 3.84 \\
\hline \multirow{3}{*}{ 1971-1990 } & \multirow{3}{*}{1} & $\mathrm{r}=0$ & 28.31 & 24.28 & $\mathrm{r}=0$ & 20.30 & 17.80 \\
\hline & & $\mathrm{r} \leq 1$ & 8.01 & 12.32 & $\mathrm{r}=1$ & 6.64 & 11.22 \\
\hline & & $r \leq 2$ & 1.37 & 4.13 & $r=2$ & 1.37 & 4.13 \\
\hline \multirow{3}{*}{ 1991-2014 } & \multirow{3}{*}{1} & $\mathrm{r}=0$ & 26.31 & 24.28 & $\mathrm{r}=0$ & 20.68 & 17.80 \\
\hline & & $r \leq 1$ & 5.63 & 12.32 & $\mathrm{r}=1$ & 5.27 & 11.22 \\
\hline & & $\mathrm{r} \leq 2$ & 0.36 & 4.13 & $\mathrm{r}=2$ & 0.36 & 4.13 \\
\hline
\end{tabular}

En los tres períodos analizados, para el estadístico traza, se rechaza la hipótesis nula de no cointegración, en favor de una relación de cointegración al nivel de significatividad del 5\%. La prueba para el estadístico del máximo valor propio nos indica también la existencia de un solo vector de cointegración al 5\% de significatividad. El análisis de ambos estadísticos nos permite concluir que exis- 
te solo una relación de cointegración al nivel de confianza del 95\% en los tres períodos de estudio: 1950-1970; 1971-1990 y 1991-2014.

Una vez asumida la existencia de una relación de cointegración al 5\% en los tres periodos de estudio, se estima la ecuación de cointegración ${ }^{7}$.

Tabla 7. Especificación de los vectores de cointegración-Hipótesis general

\begin{tabular}{ccccc}
\hline Período & $\begin{array}{c}\text { Observaciones } \\
\text { incluidas }\end{array}$ & Intercepto & $\beta_{1}$ & $\beta_{2}$ \\
\hline \multirow{2}{*}{$1950-1970$} & 21 & 6.6242 & 0.1100 & 0.0065 \\
& & & {$[6.8399]$} & {$[1.0646]$} \\
$1971-1990$ & 20 & -0.0704 & 0.0146 \\
& \multirow{2}{*}{24} & {$[-6.7117]$} & {$[3.3899]$} \\
$1991-2014$ & 24 & 0.0015 & 0.0535 \\
& & & {$[0.9122]$} & {$[5.2212]$} \\
\hline
\end{tabular}

Notas: (1) Los vectores han sido normalizados para el PBI. (2) Bi es la semielasticidad del PBI respecto a la variable i. (3) Se especifica los estadísticos t entre corchetes.

El resultado del análisis de cointegración nos lleva a aceptar la presencia de una relación de equilibrio a largo plazo entre el PIB y los flujos de capitales de corto y largo plazo, para cada uno de los períodos de estudio.

Durante el período 1950 - 1970, las variables CCP y CLP tienen signo positivo y son significativas. En cuanto a la incidencia del CCP en el crecimiento del producto se observa que hay correlación entre el incremento en las entradas netas de capital de corto plazo y el incremento en el PBI; mientras que un incremento en el CLP generó un incremento en el PBI.

Durante el período 1971 - 1990, las variables CCP y CLP son significativas; no obstante CCP tiene signo negativo, lo cual implica que puede inhibir el crecimiento. En cuanto a la incidencia del CCP en el crecimiento del producto se observa que un incremento en las entradas netas de capital de corto plazo generó una disminución en el PBI; mientras que un incremento en el CLP generó un incremento en el PBI.

Durante el período 1991 - 2014, las variables CCP y CLP tienen signo positivo y son significativas. En cuanto a la incidencia del CCP en el crecimiento del producto se observa que un incremento en las entradas netas de capital de corto plazo generó también un incremento en el PBI; mientras que un incremento en el CLP generó también un incremento en el PBI.

\footnotetext{
La ecuación de cointegración adopta la forma semilogarítmica, ya que sólo la variable dependiente o regresada (en este caso el PBI) aparece en forma logarítmica. En este modelo, es la semielasticidad del PBI respecto de la variable explicativa $i$.
} 
Si bien el test de cointegración de Johansen posibilita determinar la existencia de parámetros de cointegración o ajuste a largo plazo, la metodología del VEC ${ }^{8}$ nos permite analizar la velocidad de ajuste en el corto plazo con respecto al valor retardado en un período.

La tabla 8 muestra los resultados del VEC para los períodos 1950-1970; 1971 1990 y, 1991-2014.

Tabla 8. Estimadores del modelo VEC-Hipótesis general

\begin{tabular}{|c|c|c|c|c|c|}
\hline \multicolumn{6}{|c|}{ Período 1950 - 1970} \\
\hline \multirow[t]{3}{*}{$\Delta \mathrm{LnPBI}$} & $\lambda$ & $\alpha_{10}$ & $\alpha_{11}$ & $\alpha_{12}$ & $\alpha_{13}$ \\
\hline & -0.0339 & 0.0786 & 0.1015 & 0.0009 & -0.0004 \\
\hline & {$[-2.3932]$} & [2.6852] & [0.4079] & {$[0.7075]$} & {$[-0.7201]$} \\
\hline \multicolumn{6}{|c|}{ Período 1971 - 1990} \\
\hline \multirow[t]{4}{*}{$\Delta \mathrm{LnPBI}$} & $\lambda$ & $\alpha_{10}$ & $\alpha_{11}$ & $\alpha_{12}$ & $\alpha_{13}$ \\
\hline & -0.0051 & & 0.2464 & 0.0002 & $-3.75 \mathrm{E}-05$ \\
\hline & {$[-1.4798]$} & & [ 0.9360$]$ & [1.3829] & {$[-0.3554]$} \\
\hline & & & $1991-20$ & & \\
\hline \multirow[t]{3}{*}{$\Delta \mathrm{LnPBI}$} & $\lambda$ & $\alpha_{10}$ & $\alpha_{11}$ & $\alpha_{12}$ & $\alpha_{13}$ \\
\hline & -0.0012 & & 0.70002 & $-1.57 \mathrm{E}-06$ & $3.13 \mathrm{E}-05$ \\
\hline & {$[-3.5517]$} & & [ 5.5822] & {$[-0.2821]$} & [2.3810] \\
\hline
\end{tabular}

El coeficiente del mecanismo de corrección de errores $(\lambda)$ muestra la velocidad de ajuste de las variables ante un desequilibrio en el período anterior, en correspondencia con la relación de cointegración estable a largo plazo.

En la tabla anterior puede observarse que los ajustes del crecimiento del PBI son de aproximadamente un 0.0339 para el período 1950-1970; 0.0051 para el período 1971-1990 y 0.0012 para el período 1991-2014. Asimismo, se observa que la corrección del crecimiento del PBI ocurre más rápidamente durante el período 1950-1970.

Definida la dinámica a corto y a largo plazo de los flujos de capitales de corto y largo plazo y el crecimiento del PBI de Perú en los diferentes períodos considerados entre 1950 - 2014, se analizó la posible relación causal entre el PIB y los flujos de capital, para lo cual se aplicó el test de causalidad de Granger (caso bivariado).

\footnotetext{
8 Mediante el Vector de Corrección de Errores (VEC), se vincula el comportamiento del corto plazo con el del largo plazo. Este mecanismo incorpora las divergencias manifestadas en el corto plazo respecto a la trayectoria de largo plazo.
} 
Tabla 9. Test de Causalidad de Granger-Hipótesis general

\begin{tabular}{lccccccc}
\hline \multirow{2}{*}{ Hipótesis nula } & \multicolumn{2}{c}{ Período $1950-1970$} & \multicolumn{2}{c}{ Período $1971-1990$} & \multicolumn{2}{c}{ Período $1991-2014$} \\
& Obs. & Prob. & Obs. & Prob. & Obs. & Prob. \\
\hline CCP & LPBI & 19 & 0.0565 & 18 & 0.0044 & 19 & 0.0443 \\
LPBI & CCP & 19 & 0.4000 & 18 & 0.2606 & 19 & 0.3578 \\
CLP & LPBI & 19 & 0.1547 & 18 & 0.1661 & 19 & 0.0158 \\
LPBI & CLP & 19 & 0.4918 & 18 & 0.1064 & 19 & 0.0144 \\
CLP & CCP & 19 & 0.1310 & 18 & 0.0264 & 19 & 0.1954 \\
CCP & CLP & 19 & 0.5170 & 18 & 0.3887 & 19 & 0.8312 \\
\hline
\end{tabular}

Nota: Existe relación de causalidad cuando la probabilidad asociada al F estadístico es igual o menor a $5 \%$.

Observamos causalidad unilateral de los capitales de corto plazo sobre el comportamiento del PBI, de igual forma hay causalidad bilateral entre los capitales de largo plazo sobre el PBI y de este sobre los capitales de largo plazo.

\section{DISCUSIÓN}

El objetivo de este estudio es investigar la incidencia de los flujos de capitales en el crecimiento económico, usando las nuevas técnicas de series temporales para un período de 65 años, comprendido entre 1950 y 2014. Dicho período dividido, a su vez, en tres subperíodos: 1950-1970; 1971-1990 y 1991-2014, debido básicamente a la presencia de cambios estructurales en la economía nacional y a los cambios en el volumen y la composición de los flujos de capital analizados.

Estos cambios estructurales, sustentados en pruebas econométricas, reflejan el manejo de la economía nacional, es decir, los cambios que, en el modelo económico prevaleciente para cada uno de los períodos, se ha visto reflejado en el comportamiento de las variables económicas. En el primer período de análisis, el modelo económico prevaleciente fue el Modelo Primario exportador, tal como muy bien lo señalan Dancourt, Mendoza y Vilcapoma (1997). Siguiendo a estos autores, el modelo económico prevaleciente en el siguiente período, fue el modelo denominado Modelo de Sustitución de Importaciones y en el tercer periodo prevaleció o se retomó el Modelo Primario Exportador, como base y sustento de la economía nacional, con lo cual nos vimos como economías altamente dependientes del mercado exterior, evidentemente con cambios en los centros de gravitación mundial para nuestras exportaciones, pasando del modelo primario exportador (1950-1970), cuyo centro fue los EE.UU y sus países satelitales, a ser altamente dependientes de las economía china, en este último tramo.

El gran desempeño de la economía nacional en el primer período y en el último de análisis según Dancourt, Mendoza y Vilcapoma (1997), obedece o está 
asociado al creciente ingreso de capitales registrados sobre todo en la época de los cincuenta hasta mediados de la década del setenta.

\section{CONCLUSIONES}

El flujo de capitales o entrada de capitales a la economía nacional de acuerdo a los resultados obtenidos demuestra que su incidencia en el crecimiento del producto nacional es positivo. La influencia de estos flujos de capital ha sido reflejo del modelo económico que primaba en cada período, como ya se señaló, un modelo primario exportador en el primer periodo, un modelo de sustitución de importaciones en el segundo tramo y un tercero, nuevamente con preponderancia del mercado exterior, es decir, una economía orientada a la exportación de materias primas y altamente dependiente de sectores primarios principalmente, a lo que hay añadir una mayor diversificación en inversiones terciarias.

En el análisis individual de la incidencia de los capitales de corto plazo en la economía, es decir aquí la conclusión es que ha habido comportamientos diferenciados a lo largo del período, ello se explica por los diferentes modelos de crecimiento adoptados. En el primer período 1950-1970, hay una incidencia positiva, es decir, los CCP inciden de manera positiva al crecimiento del producto nacional; sin embargo, en los periodos siguientes 1971-1990 y 1991-2014, dicha influencia es negativa, en el sentido de que los capitales se vuelven más especulativos y obedecen a los vaivenes de la economía mundial.

La IED o inversión de largo plazo privada y la inversión pública, ha mantenido comportamientos diferenciados en los períodos de análisis: en el primer tramo, hay una influencia directa, es decir positiva pero con una repercusión bastante baja. En el siguiente período, la IED cae en su influencia sobre el crecimiento del producto. El modelo implantado no incentivaba el aumento de la IED. Sin embargo, en el tercer período, con un mayor dinamismo de la economía mundial y la entrada al mercado internacional de economías emergentes del sudeste asiático y sobre el surgimiento de la economía china, hace que la IED influya significativamente en el producto.

\section{AGRADECIMIENTOS}

A la Universidad Nacional Santiago Antúnez de Mayolo, por las facilidades financieras en la realización del presente trabajo de investigación. A los colegas del Doctorado en Economía de la Universidad Nacional Mayor de San Marcos, por sus acertados y valiosos comentarios. Los errores son de exclusiva responsabilidad del equipo investigador. 


\section{REFERENCIAS BIBLIOGRÁFICAS}

Álvarez, Agustín; Barraza, Santiago y Legato, Ana. 2009. «Inversión Extranjera Directa y Crecimiento Económico en Latinoamérica». La Serena. Vol. 20(6). Chile: Información Tecnológica.117.

Banco Mundial 2001. Global Development Finance. Washington.

Banco Central de Reserva del Perú. 2014. Cuadros anuales históricos. Lima: <http://www.bcrp.gob.pe/estadisticas/cuadros-anuales-historicos.html> [Consulta: 20-01-2016]

Boyan, Rafael y Loza Gabriel. 2005. «Flujo de capitales y Crecimiento». Bolivia: Revista de Análisis Económico. Vol. 20. Junio 2005. 5-6.

Cubillas, Julio. 2013. Términos de Intercambio, Flujos de capital y Crecimiento Económico en el Perú; un enfoque de Vectores Autorregresivos. Lima: UNI

Dancourt, Oscar; Mendoza, Waldo y Vilcapoma, Leopoldo. 1997. «Fluctuaciones económicas y shocks externos, Perú, 1950-1996». Revista Economía. Vol. XX. Lima: Departamento de Economía PUCP.39-40.

Mody, Ashoka y Murshid, Antu. 2002. Growing Up with Capital Flows. Washington: IMF Working

Presentado: 07/02/2017

Aceptado: $17 / 05 / 2017$

\section{Correspondencia}

Jorge Manrique Cáceres

j.manrique.c@hotmail.com 